\title{
Age, creatinine clearance, and ejection fraction (mACEF) score predicts long-term cardiac mortality in patients with hypertrophic obstructive cardiomyopathy treated non-invasively
}

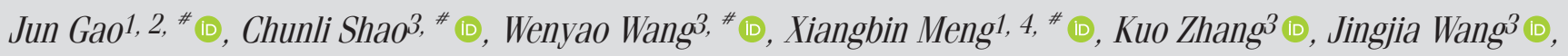 \\ Mingqi Zheng2 (1), Yi-Da Tang1 (i)
}

${ }^{1}$ Department of Cardiology, Peking University Third Hospital; Beijing-China

${ }^{2}$ Heart Center, The First Hospital of Hebei Medical University; Shijiazhuang-China

${ }^{3}$ Department of Cardiology, State Key Laboratory of Cardiovascular Disease, Fuwai Hospital, National Center for Cardiovascular Diseases,

Chinese Academy of Medical Sciences and Peking Union Medical College; Beijing-China

${ }^{4}$ Central China Fuwai Hospital; Central China Branch of the National Cardiovascular Center; Department of Cardiology,

Zhengzhou University People's Hospital; Henan Provincial People's Hospital; Zhengzhou-China

\section{ABSTRACT}

Objective: Presently, an effective model to predict long-term cardiac mortality in patients with hypertrophic obstructive cardiomyopathy (HOCM) is lacking. Therefore, the objective of this study was to evaluate the predictive value of the modified Age, Creatinine clearance, and Ejection Fraction (mACEF) score for long-term cardiac mortality in patients with HOCM.

Methods: Two hundred and ninety two patients with HOCM treated non-invasively were enrolled in this study, all of whom had intact medical information.

Results: Over a median follow-up period of 41.9 months, 28 cardiac deaths occurred. In univariate Cox regression analysis, the mACEF score was associated with long-term cardiac death [hazard ratio (HR) $=1.795,95 \%$ confidence interval (CI) 1.518-2.124, $p<0.001$ ]. Multiple Cox regression analysis identified the mACEF score as an independent risk factor for long-term cardiac death (adjusted $\mathrm{HR}=1.372,95 \% \mathrm{Cl} 1.076-1.749$, $\mathrm{p}=0.011$ ). Analysis of the receiver operating characteristic (ROC) for long-term cardiac death showed that the mACEF score had a considerable predictive value (area under ROC 0.844 , sensitivity $89.29 \%$, specificity $75.00 \%$ ) with an optimum cut-off value of 0.96 . The study population was divided into high-risk (mACEF score $\geq 0.96, \mathrm{n}=91$ ) and low-risk (mACEF score $<0.96, \mathrm{n}=201$ ) groups according to the optimum cut-off value. Kaplan-Meier survival analysis was performed and showed a dramatic higher rate of long-term cardiac mortality in the high-risk group than in the low-risk group $(27.4 \%$ vs. $1.7 \%, \mathrm{p}<0.001$ by log-rank test).

Conclusion: The mACEF score has a considerable predictive value for long-term cardiac mortality in patients with HOCM treated non-invasively. A mACEF score $\geq 0.96$ could be considered as a sign of poor prognosis in patients with HOCM.

Keywords: hypertrophic obstructive cardiomyopathy, cardiac, mortality, risk factor, prognosis

Cite this article as: Gao J, Shao C, Wang W, Meng X, Zhang K, Wang J, et al. Age, creatinine clearance, and ejection fraction (mACEF) score predicts long-term cardiac mortality in patients with hypertrophic obstructive cardiomyopathy treated non-invasively. Anatol J Cardiol 2021; 25: 691-8.

\section{Introduction}

Hypertrophic obstructive cardiomyopathy (HOCM) is one of the most common monogenetic heart diseases characterized by unexplained left ventricular wall hypertrophy and left ventricular outflow tract (LVOT) obstruction (occurring in approximately $37 \%$ patients with hypertrophic cardiomyopathy) $(1,2)$. Patients with HOCM are at increased risk of heart failure, sudden cardiac death, and atrial fibrillation with a lower life expectancy.

A rapid and effective risks stratification method plays an important role in the management of HOCM. Presently, the HCMrisk-SCD model, which is recommended by the 2014 European Society of Cardiology (ESC) guideline, is the most widely used method of sudden cardiac risk stratification in patients

\#These authors were the most frequently contributors.

Address for Correspondence: Yi-Da Tang, MD, Department of Cardiology, Peking University Third Hospital,

No. 49 Huayuanbei Road, Beijing-China

Phone: 86-10-88396171 E-mail: drtangyida@126.com

Accepted Date: 05.04.2021 Available Online Date: 20.09.2021

(C) Copyright 2021 by Turkish Society of Cardiology - Available online at www.anatoljcardiol.com DOl:10.5152/AnatolJCardiol.2021.50322 


\section{HIGHLIGHTS}

- Presently, an effective model to predict long-term cardiac mortality in patients with hypertrophic obstructive cardiomyopathy (HOCM) is lacking.

- The modified age, creatinine clearance, and ejection fraction (mACEF) score has a considerable predictive value for long-term cardiac mortality in patients with HOCM treated non-invasively.

- $\quad$ The mACEF score might serve as a tool for stratification of long-term cardiac death risk in patients with HOCM.

with hypertrophic cardiomyopathy (HCM). The HCMrisk-SCD model was designed to save lives from sudden cardiac death (SCD) by identifying patients who were at high risk of SCD and to avoid unnecessary implantable cardioverter defibrillators in low-risk patients. SCD is one of the main causes of cardiac death in HOCM; however, other causes such as heart failure and related multiple organ failure should not to be ignored. In addition, the equation of the HCMrisk-SCD model is extremely complex with detailed medical information, including family history, Holter monitoring result, echocardiogram indices, and even cardiac magnetic resonance (CMR) image being required (3). Thus, there is an urgent need for an efficient and simple way to identity patients with HOCM at high risk of cardiac death.

The age, creatinine, and ejection fraction (ACEF) score, a simple risk assessment tool, is calculated using only three variables (4) and was first developed to predict perioperative mortality in patients undergoing elective cardiac surgery. The modified ACEF (mACEF) score was remodeled using creatinine clearance instead of creatinine (5), which provided a better predictive accuracy in cardiac operations (6-8). Actually, age, creatinine clearance rate, and left ventricular ejection fraction are the three most common prognostic markers in heart diseases. Therefore, in this study, we sought to evaluate the feasibility, effectiveness, and accuracy of the mACEF score to predict cardiac mortality in patients with HOCM on non-invasive treatment.

\section{Methods}

\section{Ethics statement}

The study was conducted in full compliance with the Declaration of Helsinki and China's regulations and guidelines on clinical practice. The Ethics Committee of Fuwai Hospital approved this study (Ethics Approval \# 2015-700) with waiver of informed consent.

\section{Study participants}

This was a prospective, single-center cohort study. All the patients in this study were enrolled at the Fuwai Hospital (National Center of Cardiovascular Diseases, Beijing, China). The flowchart of inclusion and exclusion is shown in Figure 1.

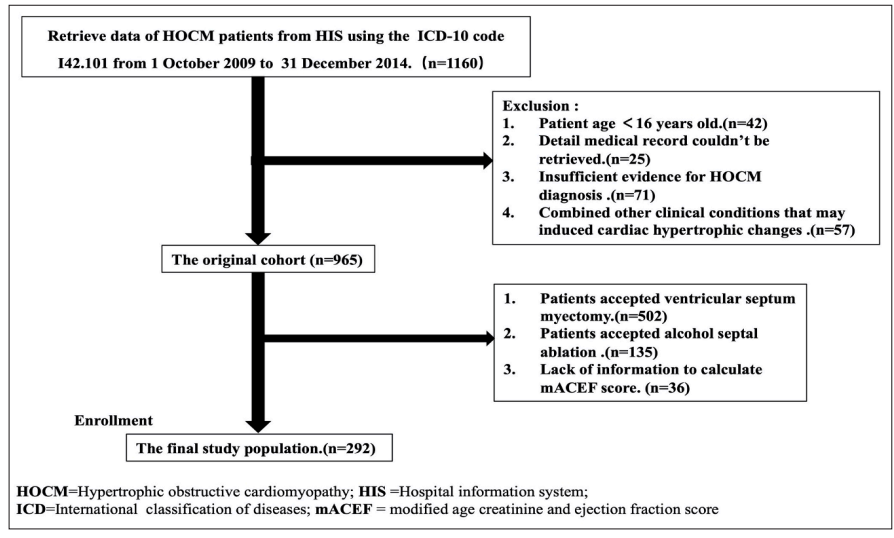

Figure 1. Flowchart of patient inclusion

A total of 965 adult patients with HOCM (age $>16$ years) were enrolled. Of these patients, 502 underwent ventricular septal myectomy, 135 had alcohol septal ablation (with two ICD implantations after the operation), and 36 patients lacked the data to calculate mACEF score; and all these patients were excluded. The remaining 292 patients with complete clinical information, medical history, and calculated mACEF score were enrolled in the study. All the enrolled patients were without any other cardiac or systemic diseases, inducing cardiac hypertrophic changes (such as uncontrolled hypertension or congenital heart disease, etc.).

\section{Data collection and definitions}

Clinical data were collected through the review of the medical records. Blood samples were obtained during hospitalization regularly. Cockcroft-Gault equation was used to calculate creatinine clearance (9). The mACEF was obtained from following equation: age/ejection fraction $(\%)+1$ point (for every 10 $\mathrm{mL} / \mathrm{min}$ reduction in creatinine clearance below $60 \mathrm{~mL} / \mathrm{min} / 1.7$ $\left.\mathrm{m}^{2}\right)(10)$.

The diagnosis of HOCM was based on the following criteria (3); wall thickness $\geq 15 \mathrm{~mm}$ in one or more left ventricular myocardial segments as measured by any imaging technique (echocardiography, cardiac magnetic resonance imaging, or computed tomography) or wall thickness 13 to $14 \mathrm{~mm}$ with family history, noncardiac symptoms and signs, electrocardiogram abnormalities, laboratory tests, and multimodality cardiac imaging; and patients with dynamic left ventricular outflow tract (LVOT) obstruction with an LVOT gradient $\geq 30$ $\mathrm{mm} \mathrm{Hg}$ at rest or during physiological provocation, such as Valsalva maneuver, standing, and exercise. Significant dynamic LVOT obstruction was documented with two-dimensional and Doppler echocardiography; or in patients in whom echocardiography was insufficient, via invasive hemodynamic catheterization with provocation. Atrial fibrillation (AF) was defined as patients with a history of paroxysmal, persistent, or permanent atrial fibrillation with documented ECG or Holter that showed AF. Ventricular tachycardia (VT) was defined as a run of $\geq 4$ consecutive ventricular premature beats documented by ECG or Holter. 


\section{Follow-up and clinical outcomes}

Follow-up began at the time of first patient clinical contact after October 01, 2009, in Fuwai Hospital. Follow-up data were collected from the record of outpatient clinic visit, phone calls, or medical records on readmission. The primary endpoint was general cardiac death. Cardiac death was defined as death because of heart failure, sudden cardiac death, cardiogenic shock, or multiorgan failure owing to cardiac causes. The unwitnessed death and death of unknown causes were also classified as cardiac death.

The patients lost to follow-up were censored at the last known contact date.

\section{Statistical analysis}

The data was analyzed using Statistical Package for the Social Sciences version 26 for MACOS and Med-Calc version 16. 8. 4. Descriptive statistics were used to summarize baseline characteristics. Categorical variables were analyzed using the chi-squared or Fisher exact tests. Normality of all variables was tested with one sample Kolmogorov-Smirnov normality test. Normally distributed continuous variables were presented as means \pm standard deviation and analyzed by student's t test, and nonnormal continuous variables were presented as median (lower quartile, upper quartile) and analyzed using Mann-Whitney $U$ test. Putative risk factors of cardiac death were identified with univariate and multiple analyses with the Cox proportional hazards model to estimate hazard ratio (HR) with $95 \%$ confidence intervals (Cls). Baseline covariates identified by univariate analysis and clinical relevance were included in the multiple analysis, and an entry criterion $p$ value of 0.1 was used. Discrimination was measured using the area under the receiver operating characteristic curve (AUROC). Comparison of ROC curve was done using DeLongs test performed by MedCalc version 16. 8. 4. Estimate of survival between high and low levels of mACEF were analyzed with the Kaplan-Meier method, and the comparison between the two groups was done using the log-rank test. A p value $<0.05$ was considered statistically significant.

\section{Result}

\section{Baseline clinical characteristics}

The baseline clinical characteristics are listed in Table 1. Of the total study population, $59.5 \%$ were men with a mean age of 54.86 years. The median follow-up time was 41.9 months. During follow-up, cardiac death occurred in $28(9.5 \%)$ patients. Patients who died were found to be older with poor renal function (higher serum creatinine and lower creatinine clearance rate). These patients also had significantly higher high-sensitivity C-reactive protein (hs-CRP) and lower hemoglobin concentration. Among echocardiogram indices, LVOT gradient and left ventricular ejection fraction (LVEF) were significantly lower and the diameter of the left atrium was higher in patients with cardiac death than in those free from cardiac death. The mACEF score was also found to be significantly higher in patients with cardiac death. In addition, higher incidences of $\mathrm{AF}$ and worsening heart function clas- sification [New York Heart Association (NYHA) functional class III or IV] were found in patients with cardiac death. However, the association between the presence of VT and cardiac death was not significant (chi-squared test, $\mathrm{p}=0.604$ ).

Modified age, creatinine clearance, and ejection fraction score and cardiac death

The association of clinical indices with cardiac death was calculated using univariate Cox regression (left part of Table 2). The mACEF score was found to be associated with long-term cardiac mortality (unadjusted $\mathrm{HR}=1.795 ; 95 \% \mathrm{Cl} 1.518-2.124$; $\mathrm{p}<0.001)$. Other 13 variables were also identified as putative risk factors for long-term cardiac mortality according to univariate Cox regression analysis. Beside age, serum creatinine, and LVEF, which were the components of the formula of $\mathrm{mA}$ CEF; all putative factors were put into the multivariate Cox regression analysis. The mACEF score remained an independent predictor for long-term cardiac mortality in HOCM ( $H R=1.372$; 95\% CI 1.076-1.749; $\mathrm{p}=0.011$; right part of Table 2). In addition, hs-CRP (HR=1.122; 95\% Cl 1.041-1.210; $\mathrm{p}=0.003$ ), presence of atrial fibrillation (HR=3.185; 95\% $\mathrm{Cl} 1.396-7.265 ; \mathrm{p}=0.006)$, and NYHA heart function classification III-IV (HR=1.563; $95 \% \mathrm{Cl}$ 1.108-2.205; $p=0.011$ ) were also found to be independent risk factors of long-term cardiac death.

Predictive value of the modified age, creatinine clearance, and ejection fraction score

ROC curve was performed to evaluate the predictive value of the score. As a result, the mACEF score demonstrated an excellent predictive value with AUROC of $0.844(95 \% \mathrm{Cl} 0.797-0.883$; sensitivity $89.29 \%$; and specificity $75.0 \%$ ), and the optimum cutoff value was 0.96 (Fig. 2). AUROC of hs-CRP, atrial fibrillation, and NYHA heart function classification III-IV was $0.764,0.639$, and 0.687 , respectively. The predictive value of mACEF score was significantly higher than that of AF and NYHA heart function IIIIV. Although statistically significance difference was not found, AUROC of mACEF was numerically higher than that of hs-CRP (0.844 vs. $0.764, p=0.215$ ) (Fig. 3).

Survival analysis of the modified age, creatinine clearance, and ejection fraction score in long-term cardiac death

The study population was divided into high-risk (mACEF score $\geq 0.96, n=91$ ) and low-risk (mACEF score $<0.96, n=201$ ) groups according to the optimum cut-off value. The Kaplan-Meier survival analysis was performed and showed a dramatic higher rate of long-term cardiac death in the high-risk group than in the lowrisk group ( $27.4 \%$ vs. $1.7 \%$, $p<0.001$ by log-rank test, Fig. 4).

\section{Discussion}

This study was the first to validate the use of mACEF score in HOCM and found a significant association between mACEF score and cardiac mortality during long-term follow-up. Our findings suggested that the mACEF score could be considered as an effective tool for risk stratification in HOCM. 


\begin{tabular}{|c|c|c|c|c|}
\hline & Free from cardiac death $(n=264)$ & Cardiac death $(n=28)$ & Total (n = 292) & $P$-value \\
\hline \multicolumn{5}{|l|}{ Demographics } \\
\hline Male, n $(\%)^{c}$ & $160(60.6)$ & $14(50.0)$ & $174(59.5)$ & 0.190 \\
\hline Age, years ${ }^{d}$ & $53.57 \pm 13.28$ & $61.11 \pm 12.05$ & $54.86 \pm 13.74$ & $<0.001$ a \\
\hline $\mathrm{BMI} d$ & $25.65 \pm 3.93$ & $24.30 \pm 3.71$ & $25.52 \pm 3.92$ & 0.080 \\
\hline Systolic BP, mm Hgd & $126.14 \pm 19.64$ & $122.48 \pm 19.90$ & $125.8 \pm 19.6$ & 0.360 \\
\hline Diastolic BP, mm Hgd & $76.39 \pm 11.67$ & $74.07 \pm 9.94$ & $76.18 \pm 11.56$ & 0.320 \\
\hline mACEF score e & $0.78(0.63,0.96)$ & $1.48(1.01,4.14)$ & $0.80(0.64,1.02)$ & $<0.001 \mathrm{a}$ \\
\hline \multicolumn{5}{|l|}{ Presence of } \\
\hline Hypertension, n (\%)c & $122(46)$ & $14(50)$ & $136(46)$ & 0.840 \\
\hline Diabetes, $\mathrm{n}(\%)^{\mathrm{c}}$ & $21(8)$ & $1(4)$ & $22(7.5)$ & 0.710 \\
\hline Hyperlipidemia, n (\%)c & $106(40)$ & $11(41)$ & $117(40)$ & 0.998 \\
\hline VHD, n $(\%)^{\mathrm{c}}$ & $39(14.77)$ & $8(28.57)$ & $47(16)$ & 0.100 \\
\hline Family history of $\mathrm{HOCM}, \mathrm{n}(\%)^{\mathrm{c}}$ & $17(6.44)$ & $1(3.57)$ & $18(6)$ & 1.000 \\
\hline Smoking, $\mathrm{n}(\%)^{\mathrm{c}}$ & $126(48)$ & $12(43)$ & $138(47)$ & 0.690 \\
\hline Alcohol consumption, $\mathrm{n}(\%)^{\mathrm{c}}$ & $84(32)$ & $7(25)$ & $91(31.1)$ & 0.530 \\
\hline Atrial fibrillation, $\mathrm{n}(\%)^{\mathrm{c}}$ & $40(15)$ & $12(43)$ & $52(17)$ & $<0.001$ a \\
\hline Ventricular tachycardia, $\mathrm{n}(\%)^{\mathrm{c}}$ & $13(4.9)$ & $1(3.5)$ & $14(4.7)$ & 0.604 \\
\hline NYHA III or IV, n (\%)c & $15(6)$ & $13(54)$ & $28(9.5)$ & $<0.001$ a \\
\hline \multicolumn{5}{|l|}{ Medicine } \\
\hline Calcium antagonist, $\mathrm{n}(\%)^{\mathrm{c}}$ & $125(47.34)$ & $10(35.71)$ & $135(46.23)$ & 0.320 \\
\hline$\beta$-blocker c & $185(70.75)$ & $22(78.57)$ & $207(70.2)$ & 0.400 \\
\hline ACEI/ARB c & $93(35.2)$ & $9(32.14)$ & $102(34.9)$ & 0.870 \\
\hline \multicolumn{5}{|l|}{ Lab test } \\
\hline Hemoglobin, g/L d & $134.74 \pm 18.53$ & $121.07 \pm 20.74$ & $133.42 \pm 19.15$ & $<0.001$ a \\
\hline Creatinine, umol/L b & $72.2(62.9,84.0)$ & $81.46(71.3,120.8)$ & $73.07(63.3,84.2)$ & $<0.001$ a \\
\hline $\mathrm{CCR}, \mathrm{mL} / \mathrm{min} \mathrm{e}$ & $98.32(78.70,116.97)$ & $63.36(41.29,79.74)$ & $94.26(73.41,115.53)$ & $<0.001$ a \\
\hline $\mathrm{hs}-\mathrm{CRP}, \mathrm{mmol} / \mathrm{L}$ e & $1.49(0.77,3.20)$ & $6.49(2.29,12.44)$ & $1.66(0.78,3.81)$ & $<0.001$ a \\
\hline $\mathrm{TC}, \mathrm{mmol} / \mathrm{L}$ e & $4.34(3.75,5.15)$ & $4.13(3.68,4.99)$ & $4.29(3.74,5.12)$ & 0.440 \\
\hline $\mathrm{HDL}-\mathrm{C}, \mathrm{mmol} / \mathrm{L}$ e & $1.07(0.89,1.27)$ & $1.02(0.83,1.15)$ & $1.07(0.87,1.26)$ & 0.120 \\
\hline LDL-C, mmol/L e & $2.65(2.02,3.26)$ & $2.59(1.86,3.17)$ & $2.65(2.02,3.25)$ & 0.560 \\
\hline \multicolumn{5}{|l|}{ Echocardiography } \\
\hline LOVT, at rest, mmHg e & $60(39,88)$ & $50(19,66)$ & $58(38,85)$ & $<0.001$ a \\
\hline LVEF, \% e & $70(65,75)$ & $63(56,70)$ & $70(65,75)$ & $<0.001$ a \\
\hline LV end-diastolic diameter, $\mathrm{mm}^{\mathrm{d}}$ & $42.08 \pm 6.42$ & $45.39 \pm 9.15$ & $42.40 \pm 6.78$ & 0.010 \\
\hline LA diameter, mm e & $39(35,43)$ & $45(39,47)$ & $40(35,44)$ & 0.002 a \\
\hline Ventricular septum thickness, $\mathrm{mm}$ e & $19(16,23)$ & $18(16,20)$ & $19(16,23)$ & 0.120 \\
\hline IVS $>30 \mathrm{~mm}^{\mathrm{c}}$ & $21(7.95)$ & $2(7.14)$ & $23(7.8)$ & 0.610 \\
\hline \multicolumn{5}{|c|}{$\begin{array}{l}\text { a } P<0.05 \text { was considered to be statistically significant. } \\
\text { bSI conversion factors: to convert creatinine to mmol/L, divided by } 1000 \text {. } \\
\text { cCategorical variables were analyzed with chi-squared or Fisher exact tests. } \\
\text { dNormally distributed continuous variables were presented as means } \pm \text { standard deviation and analyzed using student's t test. } \\
\text { eNon-normally distributed continuous variables were presented as median (lower quartile, upper quartile) and analyzed using Mann-Whitney U test. } \\
\text { BMI - body mass index; CHD - coronary heart disease; VHD - valvular heart disease; HOCM - hypertrophic obstructive cardiomyopathy; NYHA - New York Heart Association functional } \\
\text { class; mACEF - modified age, creatinine, and ejection fraction; hs-CRP - high-sensitivity C-reactive protein; ACEI - angiotensin-converting enzyme inhibitor; ARB - angiotensin receptor } \\
\text { blocker; CCR - creatinine clearance rate; TC - total cholesterol; HDL-C - high density lipoprotein cholesterol; LDL-C - low density lipoprotein cholesterol; LA - left atrial; LV - left ventricle; } \\
\text { LVOTG - left ventricle outflow tract gradient; LVEF - left ventricle ejection fraction }\end{array}$} \\
\hline
\end{tabular}




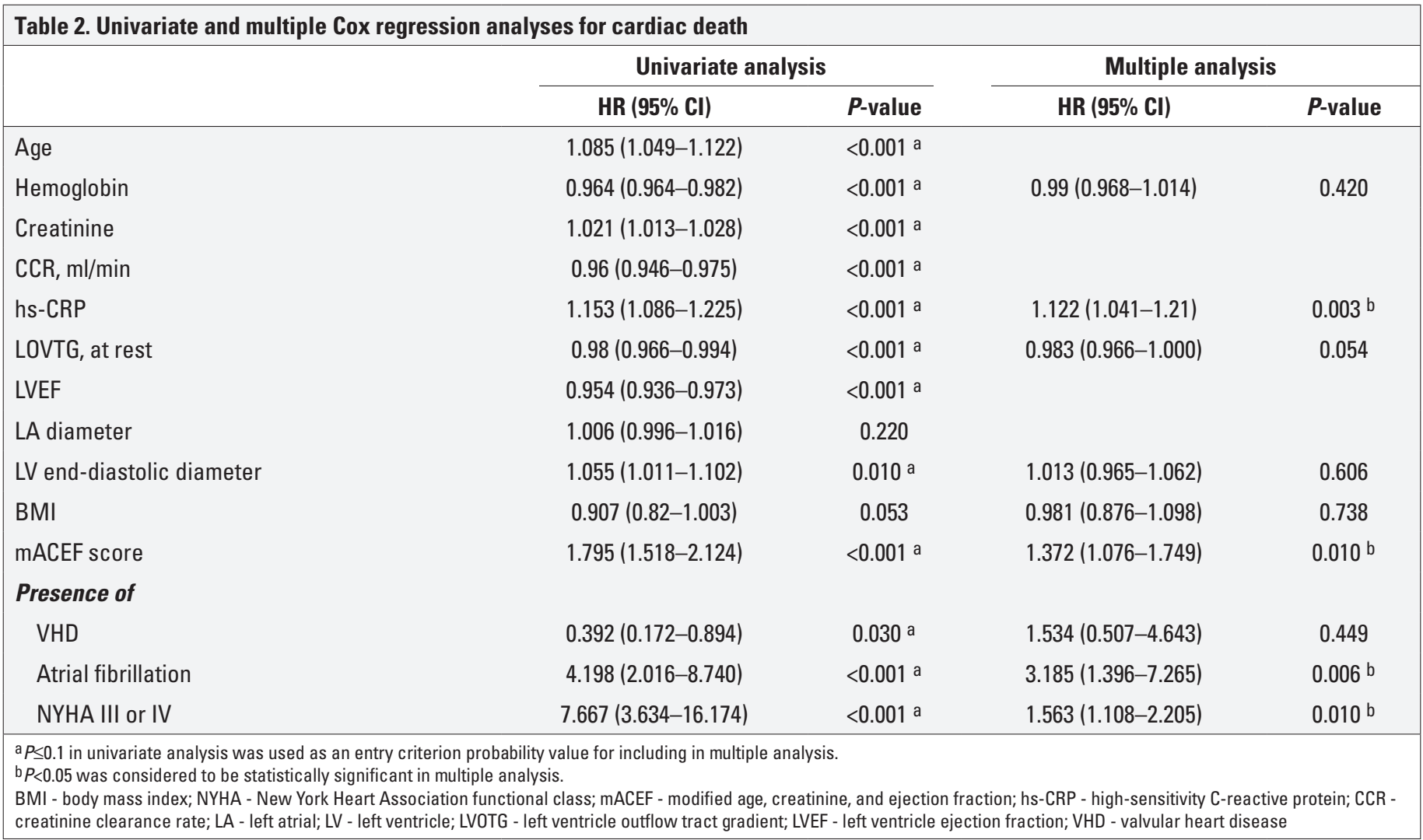

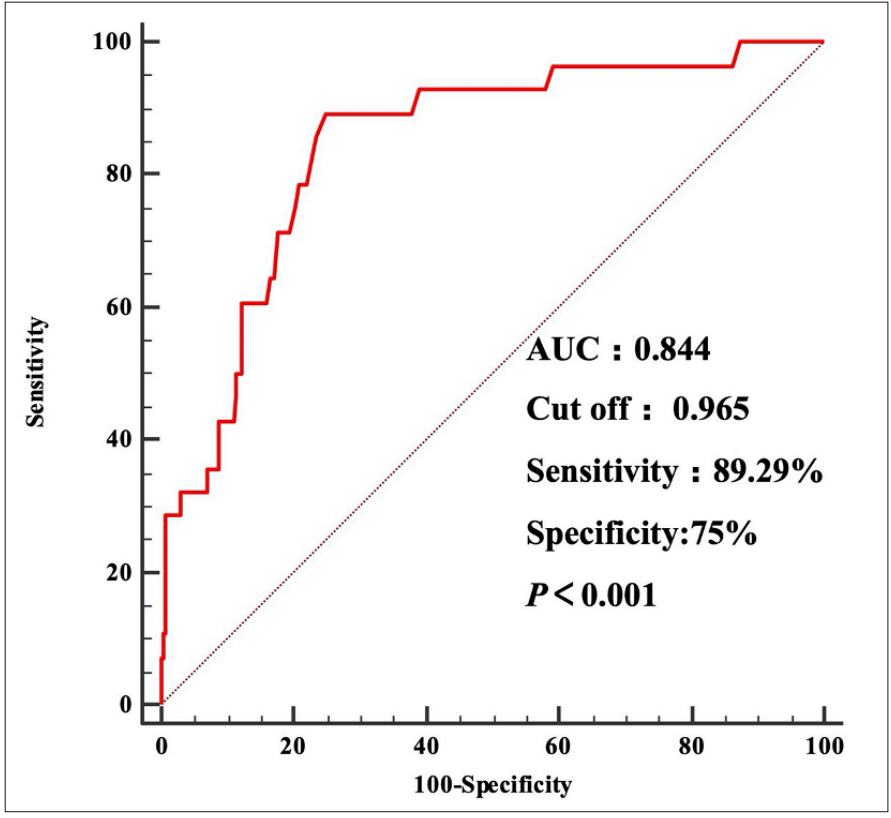

Figure 2. Receiver operating characteristic curve of the modified age, creatinine clearance, and ejection fraction score in predicting long-term cardiac death

The ACEF score was originally designed to assess the operative mortality in elective cardiac surgery with an accuracy similar or superior to pre-existing scores (additive or logistic EuroSCORE) (4). The model follows a concept of the "Occam's razor," which means "simplification" using only three factors, in- cluding age, LVEF, and preoperative creatinine. Subsequently, the ACEF score was applied to various clinical conditions (7, 11-13), and updated into different models to provide better predictive accuracy $(5,6,14,15)$. The modified ACEF (mACEF) score was remodeled from the original ACEF score by using the creatinine clearance rate as a semi-continuous variable instead of serum creatinine (5). The reason for this modification was not only because creatinine clearance rate represents a better estimate of underlying renal function but has also been previously shown to improve the predictive accuracy of cardiac risk models (16).

Reduced LVEF indicates loss of contractility and has been determined to be associated with worsening outcomes in patients with HCM (17). The age, represents the ageing of the body, was commonly used as a predictor of heart disease. In this study, age was found to be strongly associated with cardiac death. Previous studies had suggested renal function, when evaluated with creatinine clearance rate, was an independent predictor of adverse cardiac outcomes $(18,19)$. When the serum creatinine clearance was calculated using the Cockcroft-Gault equation, mathematical coupling and a co-linearity bias were introduced into the mACEF model because age in the mACEF model was counted twice, once alone and once when calculating the creatinine clearance. Therefore, we believed that the high predictive value of mACEF score in cardiac death because of HOCM would benefit from the "mutual reinforcement" between age and creatinine clearance rate.

In this study, family history of HCM in the study population was lower than in those in previous studies $(20,21)$ because 


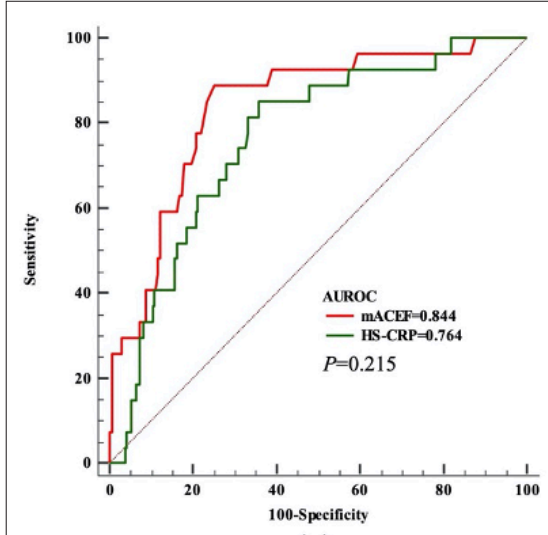

(a)

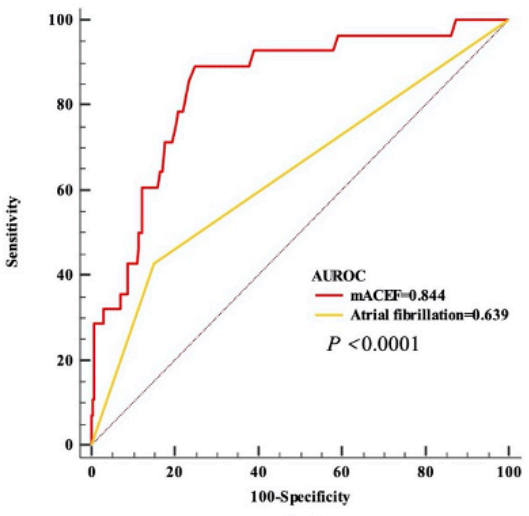

(b)

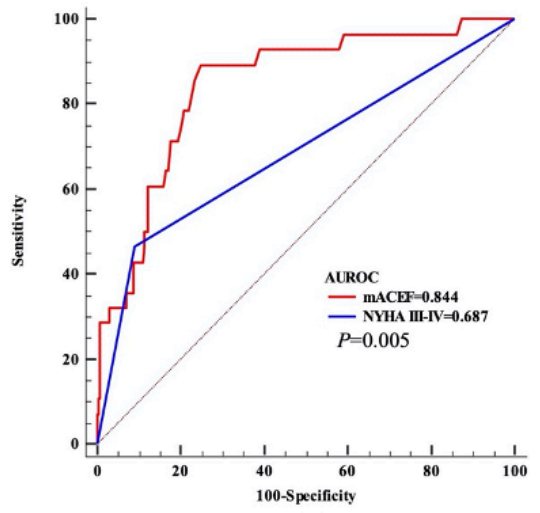

(c)

$\mathbf{m A C E F}=$ modified age, creatinine and ejection fraction score; Hs-CRP=high-sensitivity C-reactive protein; NYHA III-IV= New York Heart Association functional class III-IV

Figure 3. Pairwise comparison of receiver operating characteristic curves between the modified age, creatinine clearance, and ejection fraction score and other independent risk factors. (a) Comparison between mACEF and high-sensitivity C-reactive protein; (b) comparison between mACEF and atrial fibrillation; (c) comparison between mACEF and New York Heart Association heart function classification III-IV

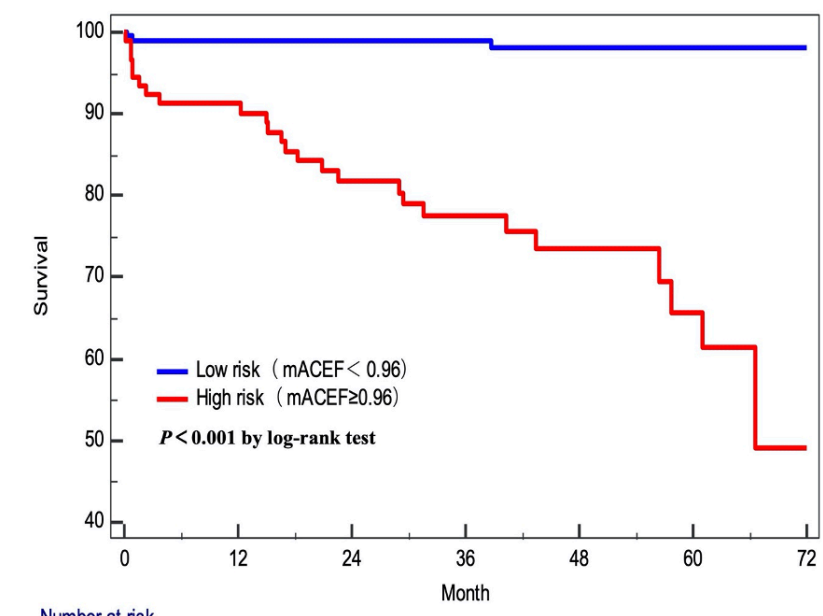

Number at risk

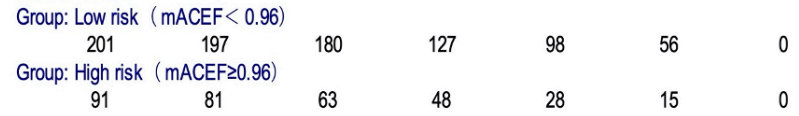

Figure 4. Kaplan-Meier curve for long-term cardiac mortality in high and low risk groups

patients could seldom recall their past family medical history in the prior generation. VT was observed in 15 patients but was not associated with cardiac mortality, the probable reason of which might be the selection bias in the study population. In this study, patients with HOCM with a high risk of sudden cardiac death and those who had undergone septal myectomy, alcohol septal ablation, and ICD implantation were excluded. This might explain why VT was not associated with cardiac mortality. We also found that the LVOT gradient was higher in patients who survived, which could be related to reduced left ventricular function. In this study LVEF, LV end-diastolic diameter, and LA diameter, though in the normal range, were found to be significantly different between patients who survived and those who suffered cardiac death. It is comprehensible that lower LVEF and higher LV end-diastolic diameter suggest poor left ventricular systolic function. LA pressure is the output of LV diastolic function. Previous studies have shown that atrial enlargement is strongly related to LV hypertrophic and systolic function (22).

In previous studies on HCM, Maron et al. (20) and Zhu et al. (23) have reported long-term cardiac mortality of $4.0 \%(0.53 \mathrm{per}$ 100 person-year) and $6.1 \%$ (1.67 per 100 person-year), which was lower than that in the present study $(9.5 \%, 2.1$ per 100 personyear). The difference may be attributed to the study population. Patients in our study were treated with medicine only to avoid the bias caused by surgical/interventional procedures. It is known that patients with HOCM treated conservatively have a higher long-term mortality $(24,25)$. This study was the first one to identify mACEF as an independent risk factor of long-term cardiac mortality in patients with HOCM treated conservatively. In addition, the presence of AF, NYHA heart function III-IV, and increasing concentration of hs-CRP were also identified as independent risk factors of HOCM, which was in accordance with previous reports $(23,26)$. The results of our study also showed that the mACEF score, in the setting of HOCM treated non-invasively, had a better prediction ability than other risk factors. Hence, the mACEF score could play a role as a stratifying tool to identify patients with HOCM patients at high risk of long-term cardiac mortality with an mACEF score $\geq 0.96$ considered to be an adverse prognostic sign.

\section{Study limitations}

This was a single center, retrospective study. Patients with HOCM treated non-invasively were enrolled, which might introduce bias and limit the generalizability. Validation of our results in other patient populations and larger sample sizes is needed. Despite the limitations, this study offers a simple indicator for risk stratification for patients with HOCM in clinical practice. 


\section{Conclusion}

The mACEF score showed a strong predictive ability for longterm cardiac mortality in $\mathrm{HOCM}$ with non-invasive treatment. An mACEF score $\geq 0.96$ could be considered as a sign of poor prognosis in patients with HOCM.

Acknowledgment: We would like to thank all my colleagues for their assistance, especially Yue-yue Xi and Shuang Gao. A special acknowledgment to Kyle Chisholm, for the helpful language polishing on this manuscript.

Institutional and financial support: This work was supported by National key research and development program (2020YFC2004700), National Natural Science Foundation of China (81825003, 91957123), CAMS Innovation Fund for Medical Sciences (CIFMS 2016-I2M-1009) and Beijing Municipal Commission of Science and Technology (Z171100000417021).

Conflict of interest: None declared.

Peer-review: Externally peer-reviewed.

Author contributions: Concept - J.G.; Design - J.G.; Supervision J.G., M.Z., Y.T.; Fundings - J.G.; Materials - J.G., C.S., W.W., X.M., K.Z., J.W., M.Z., Y.T.; Data collection \&/or processing - J.G., C.S., K.Z., J.W.; Analysis \&/or interpretation - W.W.; Literature search - J.G., C.S., W.W., X.M., K.Z., J.W., M.Z., Y.T.; Writing - J.G., C.S., W.W., X.M., K.Z., J.W., M.Z., Y.T.; Critical review - C.S., W.W., M.Z., Y.T.

\section{References}

1. Veselka J, Anavekar NS, Charron P. Hypertrophic obstructive cardiomyopathy. Lancet 2017; 389: 1253-67.

2. Maron MS, Olivotto I, Zenovich AG, Link MS, Pandian NG, Kuvin $\mathrm{JT}$, et al. Hypertrophic cardiomyopathy is predominantly a disease of left ventricular outflow tract obstruction. Circulation 2006; 114: 2232-9.

3. Authors/Task Force m, Elliott PM, Anastasakis A, Borger MA, Borggrefe M, Cecchi F, et al. 2014 ESC Guidelines on diagnosis and management of hypertrophic cardiomyopathy: the Task Force for the Diagnosis and Management of Hypertrophic Cardiomyopathy of the European Society of Cardiology (ESC). Eur Heart J 2014; 35: 2733-79.

4. Ranucci M, Castelvecchio S, Menicanti L, Frigiola A, Pelissero G. Risk of assessing mortality risk in elective cardiac operations: age, creatinine, ejection fraction, and the law of parsimony. Circulation 2009; 119: 3053-61.

5. Garg S, Sarno G, Garcia-Garcia HM, Girasis C, Wykrzykowska J, Dawkins KD, et al.; ARTS-II Investigators. A new tool for the risk stratification of patients with complex coronary artery disease: the Clinical SYNTAX Score. Circ Cardiovasc Interv 2010; 3: 31726.

6. Zhang D, Yan R, Gao G, Wang H, Fu R, Li J, et al. Validating the Performance of 5 Risk Scores for Major Adverse Cardiac Events in Patients Who Achieved Complete Revascularization After Percutaneous Coronary Intervention. Can J Cardiol 2019; 35: 1058-68.
7. Wykrzykowska JJ, Garg S, Onuma Y, de Vries T, Goedhart D, Morel MA, et al. Value of age, creatinine, and ejection fraction (ACEF score) in assessing risk in patients undergoing percutaneous coronary interventions in the 'All-Comers' LEADERS trial. Circ Cardiovasc Interv 2011; 4: 47-56.

8. Ranucci M, Pistuddi V, Scolletta S, de Vincentiis C, Menicanti L. The ACEF II Risk Score for cardiac surgery: updated but still parsimonious. Eur Heart J 2018; 39: 2183-9.

9. Cockcroft DW, Gault MH. Prediction of creatinine clearance from serum creatinine. Nephron 1976; 16: 31-41.

10. Capodanno D, Marcantoni C, Ministeri M, Dipasqua F, Zanoli L, Rastelli $S$, et al. Incorporating glomerular filtration rate or creatinine clearance by the modification of diet in renal disease equation or the Cockcroft-Gault equations to improve the global accuracy of the Age, Creatinine, Ejection Fraction [ACEF] score in patients undergoing percutaneous coronary intervention. Int J Cardiol 2013; 168: 396-402.

11. Wei XB, Wang Y, Luo JF, Hong WZ, Su Z, Zhang CX, et al. Utility of age, creatinine, and ejection fraction score in patients with type $B$ aortic dissection undergoing thoracic endovascular aortic repair. Int J Cardiol 2020; 303: 69-73.

12. Chang $\mathrm{CH}$, Lee $\mathrm{CC}$, Chen SW, Fan PC, Chen YC, Chang SW, et al. Predicting Acute Kidney Injury Following Mitral Valve Repair. Int J Med Sci 2016; 13: 19-24.

13. Wei XB, Su ZD, Liu YH, Wang Y, Huang JL, Yu DQ, et al. Age, creatinine and ejection fraction (ACEF) score: a simple risk-stratified method for infective endocarditis. OJM 2019; 112: 900-6.

14. Pyxaras SA, Mangiacapra F, Wijns W, Di Serafino L, De Vroey F, Toth $G$, et al. ACEF and clinical SYNTAX score in the risk stratification of patients with heavily calcified coronary stenosis undergoing rotational atherectomy with stent implantation. Catheter Cardiovasc Interv 2014; 83: 1067-73.

15. Denegri A, Mehran R, Holy E, Taramasso M, Pasotti E, Pedrazzini $\mathrm{G}$, et al. Post procedural risk assessment in patients undergoing trans aortic valve implantation according to the age, creatinine, and ejection fraction-7 score: Advantages of age, creatinine, and ejection fraction-7 in stratification of post-procedural outcome. Catheter Cardiovasc Interv 2019; 93: 141-8.

16. Walter J, Mortasawi A, Arnrich B, Albert A, Frerichs I, Rosendahl U, et al. Creatinine clearance versus serum creatinine as a risk factor in cardiac surgery. BMC Surg 2003; 3: 4.

17. Rowin EJ, Maron BJ, Carrick RT, Patel PP, Koethe B, Wells S, et al. Outcomes in Patients With Hypertrophic Cardiomyopathy and Left Ventricular Systolic Dysfunction. J Am Coll Cardiol 2020; 75: 303343.

18. Higuchi S, Minami Y, Shoda M, Shirotani S, Kanai M, Kataoka S, et al. Effect of Renal Dysfunction on Risk of Sudden Cardiac Death in Patients With Hypertrophic Cardiomyopathy. Am J Cardiol 2021; 144: 131-6.

19. Huang FY, Zhang JL, Huang BT, Peng Y, Chen SJ, Chen M. Renal function as a predictor of outcomes in patients with hypertrophic cardiomyopathy: A cohort study of a hospitalized population. Clin Chim Acta 2021; 512: 92-9.

20. Maron BJ, Rowin EJ, Casey SA, Link MS, Lesser JR, Chan RH, et al. Hypertrophic Cardiomyopathy in Adulthood Associated With Low Cardiovascular Mortality With Contemporary Management Strategies. J Am Coll Cardiol 2015; 65: 1915-28.

21. Zhu L, Wang J, Wang Y, Jia L, Sun K, Wang H, et al. Plasma Uric Acid as a Prognostic Marker in Patients With Hypertrophic Cardiomyopathy. Can J Cardiol 2015; 31: 1252-8. 
22. Cuspidi C, Negri F, Sala C, Valerio C, Mancia G. Association of left atrial enlargement with left ventricular hypertrophy and diastolic dysfunction: a tissue Doppler study in echocardiographic practice. Blood Press 2012; 21: 24-30.

23. Zhu L, Zou Y, Wang Y, Luo X, Sun K, Wang H, et al. Prognostic Significance of Plasma High-Sensitivity C-Reactive Protein in Patients With Hypertrophic Cardiomyopathy. J Am Heart Assoc 2017; 6: e004529.

24. Ball W, Ivanov J, Rakowski H, Wigle ED, Linghorne M, RalphEdwards $A$, et al. Long-term survival in patients with resting obstructive hypertrophic cardiomyopathy comparison of conservative versus invasive treatment. J Am Coll Cardiol 2011; 58 : 2313-21.

25. Ommen SR, Maron BJ, Olivotto I, Maron MS, Cecchi F, Betocchi S, et al. Long-term effects of surgical septal myectomy on survival in patients with obstructive hypertrophic cardiomyopathy. J Am Coll Cardiol 2005; 46: 470-6.

26. Vaidya K, Semsarian C, Chan KH. Atrial Fibrillation in Hypertrophic Cardiomyopathy. Heart Lung Circ 2017; 26: 975-82. 